\title{
Práticas de cuidado de enfermeiros à pessoa com Diabetes mellitus na hospitalização
}

\author{
Nursing care practices for people with Diabetes mellitus during hospitalization \\ Prácticas de cuidados de enfermería para personas con Diabetes mellitus durante la hospitalización
}

Caroline Rocha Batista Barcellos ORCID: https://orcid.org/0000-0003-2793-0163 Hospital Regional Hans Dieter Schmidt, Brasil E-mail: caroline.rbb@gmail.com

Juliana Graciela Vestena Zillmer ORCID: https://orcid.org/0000-0002-6639-8918 Universidade Federal de Pelotas, Brasil E-mail: juliana.graciela@ufpel.edu.br

Barbara Resende Ramos

ORCID: https://orcid.org/0000-0001-9855-5052 Universidade Federal de Pelotas, Brasil

E-mail: barbararesende.ramos@gmail.com

Caroline de Leon Linck

ORCID: https://orcid.org/0000-0001-9487-3061 Universidade Federal de Pelotas, Brasil E-mail: carollinck15@gmail.com

Samanta Frey Borges

ORCID: https://orcid.org/0000-0001-6676-8387 Universidade Federal de Pelotas, Brasil samantafrey2@hotmail.com

Fernanda Salmentão

ORCID: https://orcid.org/0000-0003-4683-7992 Universidad de La Republica, Uruguai E-mail: fersal1@hotmail.com

\begin{abstract}
Resumo
O enfermeiro tem papel fundamental na implementação de práticas de cuidado que potencializem o manejo do diabetes mellitus pela própria pessoa no hospital. Descrever as práticas de cuidado realizadas pelos enfermeiros à pessoa com diabetes mellitus na internação no hospital. Pesquisa qualitativa realizada em hospital de ensino na Região Sul do Brasil. O trabalho de campo foi desenvolvido entre setembro e outubro de 2019, com 15 enfermeiros de unidades de internação clínica, médica e de urgência e emergência selecionados mediante amostra intencional. A técnica de produção de dados incluiu a entrevista semiestruturada e, para organização e gerenciamento dos dados utilizou-se o Programa Ethnograph V6 e, analisados por meio de análise de conteúdo. Foram construídas três categorias: a primeira, Sistematização da Assistência de Enfermagem, reconheceram e foram conscientes da importância de desenvolve-la, destacando a prescrição de enfermagem como essencial para prover práticas de cuidado; a segunda, Atividades Assistenciais, principalmente, o controle e monitoramento da glicemia capilar, manejo da aplicação de insulina, monitoramento de episódios agudos de hipoglicemia ou hiperglicemia e, a terceira, Atividades Educativas, às orientações individuais e coletivas corresponderam a alimentação, uso de medicamentos, cuidados com os pés e, (re)conhecer modificações no próprio corpo. As práticas desenvolvidas visam a recuperação e promoção da saúde e, prevenção de complicações na perspectiva de promover o autocuidado à pessoa com diabetes e o preparo para retorno ao domicílio.
\end{abstract}

Palavras-chave: Diabetes mellitus; Doenças não transmissíveis; Enfermagem; Hospitais; Pesquisa qualitativa.

\begin{abstract}
The nurse has a key role in the implementation of care practices that enhance the management of diabetes mellitus by the patient in the hospital. It aims to describe the nurses' care practices given to people with diabetes mellitus during hospitalization. This is a qualitative research carried out in a teaching hospital in the southern region of Brazil. Fieldwork was carried out from September to October 2019, among 15 nurses from clinical, medical and urgent and emergency units selected through an intentional sample. The data production technique included semi-structured interviews and the Ethnograph V6 Program was used to organize and manage the data, which were analyzed through content analysis. Three categories were constructed: the first, Nursing Care Systematization, which the importance of its development was consciously recognized, highlighted the nursing prescription as essential to provide care practices; the second, Assistance Activities is about the control and monitoring of capillary glycemia, management of insulin application, monitoring of acute episodes of hypoglycemia or hyperglycemia and, the third, Educational Activities refers to individual and collective guidelines that correspond to food, use of medicines, foot care and (re) knowing the changes
\end{abstract}


that occur in the body itself. The practices developed aim at both the recovery and promotion of health and the prevention of complications in the perspective of promoting the self-care of people with diabetes and their preparation to return to their homes.

Keywords: Diabetes mellitus; Noncommunicable diseases; Nursing; Hospitals; Qualitative research.

\section{Resumen}

El enfermero tiene un papel fundamental en la implementación de prácticas asistenciales que potencien el manejo de la diabetes mellitus por parte de la propia persona en el hospital. Describir las prácticas de cuidado que realizan los enfermeros a la persona con diabetes mellitus internada en el hospital. Investigación cualitativa realizada en un hospital universitario de la región sur de Brasil. El trabajo de campo se realizó entre septiembre y octubre de 2019, con 15 enfermeros de unidades clínicas, médicas y de urgencias y emergencia seleccionados por medio de muestra intencional. La técnica de producción de datos incluyó la entrevista semiestructurada y, para la organización y manejo de los datos se utilizó el Programa Ethnograph V6 y se analizó a través de análisis de contenido. Se construyeron tres categorías: la primera, Sistematización de la Atención de Enfermería, reconocieron y fueron conscientes de la importancia de desarrollarla, destacando la prescripción de enfermería como fundamental para brindar prácticas de cuidado; la segunda, Actividades Asistenciales, principalmente, el control y supervisión de la glucemia capilar, manejo de la aplicación de insulina, supervisión de episodios agudos de hipoglucemia o hiperglucemia y, el tercero, Actividades Educativas, a las orientaciones individuales y colectivas correspondieron a la alimentación, uso de medicamentos, cuidado de los pies y, (re) conocer los cambios en el propio cuerpo. Las prácticas desarrolladas tienen como objetivo la recuperación y promoción de la salud y la prevención de complicaciones bajo la perspectiva de promover el autocuidado de las personas con diabetes y la preparación para el regreso a la casa.

Palabras clave: Diabetes mellitus; Enfermedades no transmisibles; Enfermería; Hospitales; Investigación cualitativa.

\section{Introdução}

O diabetes mellitus tipo 2 é uma doença crônica de crescente incidência e prevalência (Sociedade Brasileira de Diabetes, 2017; Flor \& Campos, 2017). O Brasil ocupa o quarto lugar no ranking de países com maior número de pessoas entre 20 a 79 anos de idade, totalizando 14,3 milhões e, com projeção para 2040 de 23,4 milhões de pessoas com a doença no País e, 642 milhões no mundo (Sociedade Brasileira de Diabetes, 2017). Está relacionada ao aumento da utilização de serviços de saúde, maiores taxas de hospitalizações, maior incidência de doenças cardiovasculares, cerebrovasculares, insuficiência renal, cegueira, e, amputações de membros inferiores (Sociedade Brasileira de Diabetes, 2017).

Trata-se de uma doença, de caráter progressivo, que pode ocasionar mudanças na vida diária das pessoas e famílias (Pesantes et al., 2018; Mwila, Bwembya \& Jacobs, 2019) e, onerosos custos aos sistemas de saúde. O hospital é um dos serviços de saúde utilizado pelas pessoas com diabetes mellitus, principalmente em decorrência de situações agudas. Trata-se de um espaço potencial para ampliar o olhar às pessoas com a doença, a fim de, implementar ações que possibilitem tanto recuperar à saúde quanto prevenir complicações e, recorrentes hospitalizações (Arruda \& Silva, 2020). Neste, o enfermeiro tem na prática de cuidado o desafio constante de planejar e, implementar ações considerando as necessidades, individuais e, coletivas, das pessoas com diabetes mellitus e, famílias a partir do contexto sociocultural que vivem.

Para o Sociólogo Pierre Bourdie "prática é o produto da relação dialética entre uma situação e um habitus, isto é, o habitus enquanto sistema de disposições duráveis é matriz de percepção, de apreciação e de ação, que se realiza em determinadas condições sociais" (Bourdie, 1994, p.19). Estudo descreve que "as práticas de saúde, são construídas por meio de vários habitus e são orientadas por uma gama de influências institucionais, familiares e referentes ao grupo social de que fazem parte" (Acioli, Kebian, Faria, Ferraccioli \& Correa., 2014, p.638). Entende-se por prática de cuidado, o conjunto de ações desenvolvidas em um momento histórico, seja por um indivíduo ou um grupo de pessoas, a partir da inter-relação deste com elementos da cultura, dos valores e, de crenças (Zillmer, Schwartz \& Muniz, 2012). O cuidado em saúde transcende as dimensões das práticas exclusivamente técnicas, ele é representado pela escuta, toque e olhar e, é nesse encontro que o enfermeiro propicia "espaço para o diálogo valorizando as histórias de vida, a crença, e a cultura de cada indivíduo" (Acioli et al., 2014, p.638) e, isso possibilita o desenvolvimento de práticas de cuidado (Acioli et al., 2014).

A literatura internacional sobre diabetes mellitus, tendo o hospital como espaço de análise, exploraram o significado do 
ensino realizado por enfermeiros à pacientes com diabetes (Castro-Meza et al., 2017), o risco para desenvolver pé diabético (Damas-Casani, Yovera-Aldana \& Seclén Santisteban, 2017); e as possíveis intervenções de enfermagem que aumentam as condutas de autocuidado (Aguayo-Verdugo \& Orellana-Yañez, 2019). No Brasil, os estudos sobre cuidados de enfermagem às pessoas com diabetes versam sobre a educação em saúde sob o olhar do enfermeiro (Arruda \& Silva, 2020), diagnósticos, resultados e intervenções de enfermagem ao idoso (Nogueira, Medeiros, Bittencourt \& Nóbrega, 2016), e, o uso de mapas de conversação em diabetes como uma estratégia educativa (Carvalho et al., 2018). Na literatura brasileira evidencia-se que há maior ênfase em estudos na atenção primária (Silva et al., 2016; Salci, Meirelles \& Silva; Teston et al., 2018).

A enfermagem tem papel fundamental na implementação de intervenções que potencializem o manejo do diabetes mellitus pela própria pessoa visando melhorar os resultados de saúde a partir de seu contexto sociocultural (Aguayo-Verdugo \& Orellana-Yañez, 2019), considerando o hospital como um espaço potencializadora da rede de saúde. No cenário brasileiro a produção bibliográfica sobre práticas de cuidado desenvolvidas pelos enfermeiros no hospital ainda é insipiente, o que leva o tema a um debate e construção necessária, com vistas a avançar e, refletir acerca do que está sendo desenvolvido por esses profissionais e o que pode ser fortalecido. Diante do apresentado, o estudo teve como objetivo descrever as práticas de cuidado realizadas pelos enfermeiros à pessoa com diabetes mellitus na internação no hospital.

\section{Metodologia}

Estudo qualitativo realizado em um hospital de ensino na Região Sul do Rio Grande do Sul, Brasil; sendo este escolhido por manter vínculo com o Centro de Referência às pessoas com diabetes, referência na Região e, ambos estarem sob a responsabilidade de uma Universidade Pública. O trabalho de campo foi desenvolvido entre setembro a outubro de 2019, pela primeira autora, discente de enfermagem, devidamente capacitada e, sob supervisão e orientação da segunda autora que apresenta experiência nessa área do conhecimento e abordagem de pesquisa.

Os participantes do estudo foram enfermeiros de unidades de internação, mediante amostragem do tipo intencional; selecionados a partir dos seguintes critérios, ser enfermeiro da unidade há mais de seis meses e, que tivessem vivenciado à assistência à pessoa com diabetes mellitus. Como critérios de exclusão, enfermeiros em licença saúde ou férias. Para obter o total de enfermeiros nas referidas Unidades a pesquisadora contou com o apoio da gerência de enfermagem da instituição mediante o fornecimento de uma lista contendo os que estariam atuando no período da coleta, contemplando os três turnos de trabalho. Havia um total de 30 enfermeiros nas referidas Unidades, sendo que destes participaram do estudo 15 enfermeiros que atenderam os critérios de inclusão, sendo o tamanho da amostra e finalização da coleta de dados determinado pelo critério de saturação de informações (Minayo, 2017), na medida em que a informação coletada se repetia, possibilitando a construção de esquema teórico para responder à pergunta e objetivo de pesquisa.

A técnica de coleta de dado incluiu a entrevista semiestruturada, sendo elaborado um guia contendo perguntas abertas e fechadas considerando o objetivo do estudo. As entrevistas foram desenvolvidas individualmente, em horários previamente definidos com os participantes, com uma duração de 30 a 50 minutos. A entrevista contou com uma primeira pergunta aberta "Podes me contar sobre a sua experiência ao cuidar de pessoas com diabetes durante a hospitalização?", e posteriormente seguiuse com outras perguntas, por exemplo, “o que você faz para cuidar de pessoas com diabetes?”. Nas perguntas abertas da entrevista foram utilizadas perguntas que possibilitassem aprofundar o tema. Os participantes foram entrevistados em uma sala reservada do hospital, conforme escolha do participante. As 15 entrevistas foram gravadas e, transcritas na íntegra pela primeira autora, sendo posteriormente revisadas pela mesma, com escuta dos áudios gravados e leitura do texto escrito.

Para organização e gerenciamento do conjunto de dados das entrevistas foi utilizado o Programa Ethnograph V6. Este conjunto foi analisado mediante análise de conteúdo (Bardin, 2016). A análise consistiu na leitura, por parte da primeira autora, dos dados a fim de atingir a imersão e, obter uma compreensão do todo. Posteriormente, procedeu-se à leitura linha a linha, até 
geração de códigos. Em seguida, os códigos foram comparados e, fragmentos de texto selecionados para identificação dos temas, que posteriormente deram origem a categorias. Foram construídos 24 códigos, e estes deram origem a três categorias. Durante este processo, identificou-se temas relacionados às práticas de cuidado, sendo uma delas apresentadas neste artigo.

A qualidade dos dados foi garantida mediante um processo constante de reflexibilidade da primeira autora, juntamente com a supervisão e orientação da segunda autora. A pesquisa foi aprovada pelo Comitê de Ética em Pesquisa de uma universidade pública sob Parecer de número 538.882, sendo seguidos preceitos éticos da Resolução 466/12. Os participantes assinaram o Termo de Consentimento Livre e Esclarecido e, o anonimato foi garantido mediante uso de pseudônimos.

\section{Resultados}

A partir da análise dos dados foi possível conhecer e descrever as práticas de cuidado desenvolvidas por enfermeiros às pessoas com diabetes mellitus, durante a internação no hospital possibilitando ampliar e aprofundar a reflexão sobre essas práticas nesse cenário. Foram construídas três categorias sendo elas, Sistematização da Assistência de Enfermagem; Atividades Assistenciais e, Atividades Educativas.

\section{Sistematização da Assistência de Enfermagem}

A sistematização da Assistência de Enfermagem (SAE) apresentou-se como uma prática de cuidado desenvolvida pelos enfermeiros no hospital. Eles associaram a SAE à oportunidade, principalmente, de conhecer as condições físicas e emocionais do paciente com diabetes, o contexto sociocultural e, relações familiares, de ouvir e estabelecer vínculo, identificar as necessidades de saúde, monitorar o estado de saúde, e mediante ela desenvolver e direcionar intervenções de enfermagem considerando o que é prioridade em saúde. Para os participantes por meio da SAE há como se considerar que cada paciente é "único", tem sua individualidade, o que é necessário para realização do cuidado no hospital e, posteriormente possam ser condizentes com contexto do indivíduo e família e aplicadas quando retornarem ao domicílio. Tais achados podem ser identificados nos seguintes excertos:

É difícil de falar generalizando. Ah, a principal, o principal cuidado do paciente diabético, ponto, é X. É impossivel te dizer isso. Porque o paciente na verdade ele tem tempo de diabetes, ele tem outras comorbidades associadas ou não, ele tem um cuidado prévio ou não. Então tudo isso influencia na linha de cuidado. [E09]

Quando fazer o levantamento dessas informações também ter em mente de qual a realidade desse paciente. Que também não adianta a gente investir numa prescrição, numa orientação, com muitas alimentações que ele não tem condições [de comprar]. Saber qual a realidade dele, se ele tem alguém em casa que possa ajudar, se ele realmente consegue usar essas medicações sozinho, tem muitos pacientes que não conseguem, eles mesmo realizar o tratamento. Muitos saem daqui [tem alta hospitalar] sem condições, por mais que tu coloque, anote [a orientação]. [E015]

O momento da admissão do paciente com diabetes mellitus no hospital foi considerado fundamental pelos enfermeiros para planejar as práticas de cuidado. É neste momento que os enfermeiros consideraram ser o "ponto de partida", em que realizaram a "anamnese" investigaram a história de saúde, se possui diagnóstico e, ou se tem histórico familiar de diabetes, e, quais medicamentos faziam uso, além de buscar informações sobre hábitos alimentares e, de como era o dia a dia no domicílio. Estas informações coletadas foram registradas no prontuário do paciente, compartilhando com os demais profissionais e, quando necessário retornaram a consulta-las. A partir da anamnese, elegeram o prioritário, direcionando as intervenções para a alimentação e de controle da glicemia capilar. Isso pode ser evidenciado nos excertos a seguir:

Saber se ele é diabético ou se ele não é diabético, se tem histórico familiar de diabetes porque, as vezes, algumas alterações, são causadas [pelo diabetes], e as vezes o paciente nem sabe se é diabético. [E02] 
Nas nossas admissões a gente já tem como questão do paciente ser ou não diabético e, sendo ele diabético a gente já toma um cuidado maior, no sentido de estar sempre fazendo os HGTS, de 6 em 6 horas ou de 4 em 4 horas, geralmente é de 6 em 6. E dessa forma a gente consegue regular bem. [E06]

Quando o paciente interna, a gente faz uma a anamnese, e a gente já procura saber se ele tem essa patologia, e, a gente já detecta que ele faz uso de medicamento, já usa medicação para diabetes. A gente já solicita uma dieta para ele, e, geralmente é uma dieta adaptada, para ele. Ah, nós também perguntamos para ele como é o dia- dia [no domicílio].[E07]

É na admissão, que além da anamnese realizam o "exame físico" dos pacientes com diabetes mellitus. Mediante ele, buscam avaliar a "fisionomia do paciente", o peso corporal, considerando alterações físicas, como emagrecimento ou sobrepeso. Além de, direcionarem “o olhar" para possíveis complicações da doença. Entretanto, nenhum dos participantes mencionou a realização do exame do "pé diabético" Tais achados podem ser evidenciados nos seguintes excertos:

Procuro ver a fisionomia do paciente, em relação ao exame físico, ah, vejo peso, como é que tá. Também se o paciente está mais emagrecido, se ele já está mais obeso. Essas questões assim, que é o que a gente pode fazer no ambiente hospitalar. [E07]

A gente colhe todo o histórico e faz o exame físico no paciente para poder fazer essa identificação. Evidente que o paciente, por exemplo, que tem como doença crônica o diabetes e internou digamos por uma pneumonia, ah, a gente vai fazer a, durante a anamnese, o exame físico vai tentar identificar vasculopatias, neuropatias, então, tudo associado ao diabetes. [E09]

Entre as necessidades humanas básicas, os enfermeiros destacaram, que os pacientes com diabetes apresentaram como principais alterações relacionadas ao, emocional, conforto, privacidade e alimentação. Estas foram descritas como prioritárias para desenvolver as intervenções, conforme os seguintes excertos:

Aqui na unidade assim, principalmente, que vejo como prejudicado é o conforto. Eles ficam muito prejudicados. [E01]

Eu entendo a necessidade humana básica como tudo aquilo que ele precisa o necessário para ele ficar bem, comer, dormir, se alimentar bem, ter o tratamento adequado. Então para mim tudo isso faz parte, para ele ter um bom atendimento hospitalar. E o diabetes, as vezes, gera muita necessidade emocional por conta de todo esse manejo que as vezes não é adequado. [E03]

Nos diálogos com os enfermeiros foi possível identificar que eles reconheceram e foram conscientes da importância de desenvolver a SAE, destacando a prescrição de enfermagem como essencial para prover práticas de cuidado. Contudo encontraram obstáculos para desenvolvê-la, sendo o principal o sistema de informação. Este foi associado a dificuldade em prescrever cuidados; de possuir possibilidades insuficientes para elencar diagnósticos que correspondessem a diabetes; assim como a forma de apresentação das prescrições. Entre as limitações esta o fato de não poder prescrever o controle de glicemia 
capilar, com aprazamento, se necessário, considerando que além do monitoramento fixo, há situações de urgência em que deve ser realizado; além de não conter cuidados específicos para aqueles que internaram em decorrência de pé diabético. Isso pode ser constatado nos excertos a seguir:

Eu acho que poderia ser melhor, que a gente poderia já ter um diagnóstico específico né!. Para pacientes com diabetes, hipertensão e, no momento a gente não tem. Até isso foi um questionamento nosso dos enfermeiros que a gente pudesse melhorar esse sistema. Porque a gente faz tudo pelo sistema, e, é bem diferente do livro, se tu pegar ali o diagnóstico da Nanda, tu vais ver que já tem os cuidados específicos, para cada patologia, e no momento não tem isso. [E07]

A prescrição de cuidados a esses pacientes específicos, no nosso sistema de prescrição, é bem difícil. Ele não é lógico assim, não é um sistema lógico, que imediatamente vai te abrir uma linha de procedimentos e, não te abre um leque também de opções de tu colocar aquele cuidado específico, então tu acabas tendo que colocar algo meio aproximado. $E ́$, então não tem uma, a possibilidade colocar o cuidado que a gente quer, exatamente, o tipo de cuidado que a gente quer não, que é necessário ao paciente, então tem que colocar algo aproximado que muitas vezes vai destoa do diagnóstico de enfermagem, entende!? Não é aquele diagnóstico de enfermagem, é outro diagnóstico, mas o cuidado em si ele é o mais próximo do que a gente precisa para aquele paciente. Então é algo que acaba confundindo inclusive o restante da equipe para o cuidado, então acho que o sistema tem muito a melhorar. [E09]

E quanto ao diabético a gente tem uma certa dificuldade mesmo assim, por exemplo, de prescrever para verificar o HGT, conforme a pessoa fala que não tá bem, entendeu.? Para verificar o HGT, não tem essa prescrição do se necessário, só tem manhã, tarde e noite - o contínuo, e não tem esse "se necessário". Na pele tem até, mas não relacionado ao diabetes em si, tem em relacionado o geral para evitar úlcera de pressão, mas eu acho que a prescrição de enfermagem não está muito boa, mas não só para o diabético, para todos os pacientes em geral. [E013]

\section{Atividades Assistenciais}

As atividades assistenciais foram identificadas como importante prática de cuidado dos enfermeiros no hospital, principalmente, o controle e monitoramento da glicemia capilar, manejo da aplicação de insulina, monitoramento de episódios agudos de hipoglicemia ou hiperglicemia. Para os enfermeiros o monitoramento e controle da glicemia é um cuidado prioritário para pacientes com diabetes. A partir deste monitoramento direcionaram intervenções, seja mediante, uso de insulina, modificação de horários para monitoramento e ou, a implementação de esquema de medicamentos e, adequação na dieta. Isso pode ser constatado nos excertos a seguir:

O cuidado dá gente é basicamente esse, é ficar sempre verificando os HGTs nos horários certos, sempre orientando, sempre fazendo insulina, sempre fazendo esquema. Porque as vezes ele tem insulina nos horários fixos, ou metformina, mas ele tem um esquema que se aquela medicação não foi adequada, tem um esquema que a gente tem que fazer das insulinas e seguir a risca aquele esquema, acompanhar de novo HGT, explicar tudo ao paciente. [E03]

A glicemia dele é fundamental, por exemplo, se ele diz naquele dia que não se alimentou, "ah enfermeira hoje eu estava com inapetência e, não comi nada o dia todo", ai você já fica preocupada com esse paciente e faz até, liga para o plantão e, faz até HGTS extras, para saber se a gente precisa fazer uma ampola de glicose, alguma coisa, porque oscila muito rapidamente né a glicemia deles. [E012] 
Os enfermeiros apresentaram preocupação com o manejo e administração da insulina, considerando preparo e rodízio do local de aplicação a fim de evitar complicações. Diante disso, realizaram a supervisão e avaliação do rodízio mediante exame físico diário. Outro ponto descrito foi o monitoramento de episódios agudos de hipoglicemia ou hiperglicemia, em que, mediante, o hemoglicoteste avaliaram se deveriam administrar o esquema de insulina prescrito ou se necessitariam contatar o profissional médico de plantão. Associaram a realização desse teste, avaliar sinais e sintomas que indicassem alguma alteração e, consequentemente complicações irreversíveis, e até mesmo a morte. Tais achados são identificados nos seguintes excertos:

Também tem que ficar cuidando se os meninos [técnicos de enfermagem] estão fazendo rodízio na hora de fazer a aplicação subcutânea. Então é um olhar diferenciado que a gente tem que ter com o paciente diabético. [E012]

Quando a gente verifica as taxas de glicose do paciente que é diabético e, às vezes dá muito alterado ou muito abaixo do normal, tipo aí, ele está com uma quantidade grande de insulina, às vezes eles tem a insulina NPH que é de liberação lenta. Então normalmente a gente acaba entrando em contato com o plantão para saber se realmente a gente administra, porque eles podem ter uma taxa, uma queda grande na taxa da glicose deles assim. [...]. Então na verdade a gente monitora essa parte assim, a verificação da taxa de glicose, as medicações que ele tem que fazer uso e sempre atento se está muito baixa, se está muito elevada, e até atento assim aos sinais que eles apresentam. Tem sudorese, eles ficam com vertigens, as vezes até se queixam dessas coisas assim, ou até, às vezes ficam muito sonolentos. Então a primeira coisa que a gente desconfia que pode ter alguma alterada, a gente está sempre monitorando esse paciente. Atentar aos sintomas que eles podem vir a apresentar, tipo fica monitorando o sensório, verificar os sinais vitais deles e controle de HGT, essas coisas assim. [E014]

A gente fica muito atento a sensório, a todos os sinais do paciente porque muitas vezes não é o horário, de e a gente ter que fazer HGT. Então paciente que está com qualquer sintoma de hipoglicemia ou hiperglicemia que a gente perceba, a gente já realiza o HGT e já está, intervindo junto com o plantão. [E05]

Os participantes apontaram que eles têm consciência de que o paciente com diabetes tem maior risco para desenvolver lesões cutâneas no hospital em decorrência das especificidades da doença. Dessa forma, há maior preocupação em monitorar e avaliar a pele destes pacientes diariamente, iniciando com uma "avaliação" já na admissão. As práticas com o cuidado da pele e pés, relacionaram-se, tanto para prevenir lesões cutâneas quanto para tratá-las, por exemplo, sendo a mais frequente "pé diabético". Ainda, descreveram que a complexidade do cuidado se torna maior quando este já possui alguma lesão, pois o processo de cicatrização ocorrerá em maior tempo, resultando em outras complicações decorrentes do período de internação e a exposição a agentes infecciosos no hospital. Associam-se a essa recuperação a necessidade de implementação de cuidados com a alimentação e tratamento medicamentoso.

Para prevenir lesões cutâneas em pacientes com diabetes os enfermeiros utilizaram hidrantes para proteger e fortalecer a pele, mudança de decúbito e reposicionamento, principalmente dos pacientes que estavam restritos ao leito, além de intensificar orientações para reduzir o risco de quedas, como manter a grade no leito, utilizar calçado apropriado e, certificar-se da capacidade de deambulação sem auxílio e, ou solicitar apoio da equipe de enfermagem quando necessário. A Escala de Braden era utilizada em algumas unidades sendo descrita como importante ferramenta para prevenir lesões cutâneas nestes pacientes.

Quando abre uma lesão em paciente diabético a gente já pensa, meu Deus! Vai demora de cicatrizar, vai, porque 
infelizmente é uma das características do paciente diabético. Então a gente tenta já evitar que se abra lesões, já dá um cremezinho lanete, para hidratar a pele, para fortalecer aquilo ali, mas nem sempre é possível. As vezes abre de qualquer jeito, então também tem esse cuidado especial assim com as lesões. Na RUE X já tem uma escala [Escala de Braden] que ela avalia, possíveis lesões que possam aparecer no paciente quando ele interna. Você olha ele dos pés a cabeça e, anota lá na Escala de Braden. Até dezembro essa Escala vai estar implantada em todo hospital. [...]. Se ele for diabético ele já tem um risco maior de desenvolver uma lesão, se ele já vem como uma lesão a gente já vai avaliar o grau dessa lesão que ele internou, para quando ele sair a gente, [avaliar] melhorou? Piorou? [E012]

Porque as vezes o paciente está aqui e fica tempo, com uma permanência maior, ou se o paciente vai para o cirúrgico, também, paciente cirúrgico, tenho uma preocupação um pouquinho maior pelo risco de sangramento, porque ai tem as questões das feridas (...). Eu tenho uma preocupação a mais sobre a infecção nas feridas, nas feridas operatórias, então, quando o paciente é diabético e, é cirúrgico eu me preocupo com ferida. Questão de lesões e paciente cirúrgicos são as feridas após, por causa que é muito comum infectarem após. [E05]

Para os pacientes que já internaram com alguma lesão cutânea em decorrência do diabetes os enfermeiros descreveram que o cuidado era "redobrado", realizavam a evolução da lesão mediante a troca dos curativos, e, também intensificavam cuidados como mudança de decúbito e reposicionamento. Descreveram que a instituição possui um grupo de pele e, este foi considerado fundamental para avaliar e, prescrever coberturas influenciando diretamente na recuperação e tratamento das lesões. Porém, o fato de haver o referido grupo, não os eximiram de avaliar, monitorar e manejar o cuidado mediante a realização dos curativos.

Eu acho que tem bastante [dificuldades], mais dificuldades, principalmente quando o paciente já tem alguma lesão, eu acho que a dificuldade é bem maior porque a cicatrização é bem mais demorada. Então o cuidado é redobrado, tanto da função da alimentação, a função da medicação, e os curativos que precisam ser feitos. [E010]

Porque a gente cuida basicamente, quando o paciente tem curativo. Então a gente acaba cuidando dessa parte da troca de curativos dele, apesar de serem curativos que são monitorados pelo grupo de pele, muitas vezes tem a necessidade de trocar. A noite somos nós que trocamos também, ter cuidado com os curativos deles. [E014]

\section{Atividades Educativas}

As atividades educativas também foram identificadas como importante prática de cuidado dos enfermeiros no hospital, que se relacionaram, principalmente, às orientações individuais e coletivas realizadas ao paciente com diabetes mellitus e familiares. Foram realizadas por meio de conversas individuais tanto para pacientes quanto para familiares, e, que corresponderam a alimentação, uso de medicamentos, cuidados com os pés e, (re)conhecer as modificações no próprio corpo.

As orientações quanto a alimentação foi descrita pelos participantes como "informações de forma geral", pontuando a importância de manter uma alimentação saudável. Entre as orientações relacionadas a alimentação os enfermeiros reforçam diariamente a importância de os pacientes somente consumir a "dieta" fornecida pelo hospital. Essa orientação, para os enfermeiros é um desafio a ser seguida pelos pacientes, uma vez que, eles "trazem o que querem de casa", resultando, por exemplo, no aumento da glicemia capilar.

Identificou-se o controle da alimentação como sendo primordial, associado ao monitoramento de sinais e sintomas. Entre as formas de monitoramento estava a glicemia capilar, como central na realização dos cuidados. Além disto, durante a 
internação, os enfermeiros questionaram o paciente e familiar, a fim de compreender o que está ocorrendo e, como estava a alimentação. A equipe de nutrição foi apontada como referência para os enfermeiros das unidades de internação quando necessitaram fornecer orientações específicas referente a substituições e exclusão de alimentos. Tais achados são evidenciados nos excertos:

Ele ter controle com as comidas, principalmente, e ficar atento a qualquer alteração do seu corpo, seja na madrugada, quando ele tiver alguma alteração. Enfim, são vários questionamentos e orientações que a gente dá, vai depender muito do paciente em sí, da orientação dele também. [E04]

Sempre que o paciente vem com diagnóstico de diabetes a gente cuida muito a função da alimentação dele, o que ele come, o que deixa de comer. A prescrição médica com relação a isso também e, a prescrição da nutrição, a gente cuida isso também, porque muitas vezes ele se descobre.[E010]

A família foi descrita pelos enfermeiros como a responsável por trazer os alimentos de casa. Somado a isso descreveram que para orientá-los era necessário conhecer os hábitos dos pacientes e, isso demanda tempo e dependia do vínculo e interação construída. o que pode ser identificado no excerto a seguir:

Então, assim, dentro da unidade que mais utilizo, a informação que mais passo, principalmente quando ele já internou é que só coma a comida do hospital, porque tem a questão toda dos hábitos alimentares que os pacientes descompensam às vezes, porque tem toda uma situação de causa social, de, as vezes não é o paciente que faz a comida em casa, éo familiar. É toda uma questão, de vínculos e, social, de estrutura do familiar, do paciente, então, a gente sempre pede que tira, que coma somente a comida do hospital enquanto tá aqui, porque a gente demora as vezes pra perceber todos os hábitos do paciente. [E05]

As orientações quanto ao uso de medicamentos, os participantes consideraram importante identificar previamente os medicamentos que os pacientes faziam uso no domicílio, a quantidade e o horário de uso, diferenciando com o estar no hospital. Além, de manter o paciente informado quanto aos horários de administração, a finalidade e função do medicamento no organismo e as complicações do não uso, assim como uma forma de "cobrar dos profissionais" em caso de atrasos na administração.

Nós colocamos as identificações, quando precisa fazer a medicação [depois em casa], qual é o horário que é, ou se é, a mesma coisa que eu fazia na atenção básica, eu cheguei já a fazer aqui, de colocar sol e lua, sabe?. [E05]

A administração dos medicamentos também costumo orientar para que ele preste atenção. Eu acho importante o paciente saber o horário da administração dos medicamentos, o horário que acontece aqui dentro porque dai tu podes cobrar, ou perguntar, ou tirar alguma dúvida a respeito, interagir junto com a equipe, isso eu acho importante. [E010]

Entre as orientações para os cuidados com os pés estavam, principalmente, a avaliação e orientação quanto ao uso de calçado fechado e adequado, a utilização de meias confortáveis que não afetassem a circulação, corte das unhas e, secar entre os dedos após o banho. Além de manter membros inferiores elevados e, estímulo a deambulação para aqueles que tinham condição de fazê-la. 
Então, em função de ver a circulação, de ver as unhas. Porque aqui a gente tem, se o paciente é mais acamado, a gente tem todo protocolo de prevenção de úlcera de pressão. Se o paciente deambula, a gente pede, para eles caminharem mais, para ele, para circulação melhorar, que eles podem fazer aqui dentro do hospital. Tomar banho, secar bem entre os dedos dos pés, secar bem as unhas, ter cuidados, usar meias confortável, meia que não corte a circulação. [...] Manter elevado [membros inferiores] quando possivel, um travesseiro, um cobertor em baixo para não ficar encostando tanto o calcâneo, principalmente. São cuidados que a gente pode orientar enquanto estão aqui, para eles fazerem aqui. [E011]

\section{Discussão}

As práticas de cuidado realizadas pelos enfermeiros aos pacientes com diabetes na hospitalização transcorreram pela recuperação e promoção da saúde, prevenção e controle de complicações, e, orientações quanto a realização de técnicas para o tratamento e monitoramento de sinais e sintomas. A sistematização da assistência de enfermagem foi descrita como prática de cuidado fundamental para qualificar a assistência aos pacientes com diabetes mellitus no hospital, sendo ela o ponto de partida para planejar, implementar e, avaliar o cuidado. A partir dela, foi possível ampliar o olhar para além dos aspectos biológicos, olhar as dimensões emocional, social e cultural, considerando para isso se aproximar do contexto sociocultural para planejar o retorno desse paciente e familiar ao domicílio. Entretanto, vivenciaram dificuldades na implementação, relacionadas a estrutura e funcionamento do sistema de informação.

A legislação da Enfermagem Brasileira normatiza que a sistematização da assistência de enfermagem é uma prática privativa do enfermeiro, com o propósito de sistematizar o planejamento, execução, controle e avaliação dos cuidados. Trata-se de um método que compreende o histórico de enfermagem, diagnóstico de enfermagem, planejamento, implementação e avaliação de enfermagem (Cofen, 2009).

A SAE ganha relevância no cenário do cuidado por possibilitar identificar as necessidades prioritárias de saúde com a finalidade de recuperar, reabilitar e, promover à saúde de indivíduos e famílias. As necessidades prioritárias em pacientes com diabetes estão relacionadas às psicobiológicas, oxigenação, eliminação, cutâneo mucosa, sono repouso, e cuidado corporal, além de necessidades psicossociais, como o conhecimento deficiente sobre o problema de saúde (Nogueira, Medeiros, Bittencourt \& Nóbrega, 2016). Entretanto, obstáculos podem ser vivenciados pelos enfermeiros para implementação da SAE, entres eles, tempo e criatividade para elaborar estratégias para sistematizar o cuidado, seja por sistema informatizado ou não (Soares, Resck, Terra \& Camelo, 2015). Destaca-se que há a necessidade de um movimento e articulação dos enfermeiros com os gestores do hospital a fim de desenvolver estratégias que possibilitem a implementação, qualificação do sistema de informatização e, capacitações para os profissionais promovendo a construção do raciocínio clínico subsidiado em referencial teórico que fundamente as práticas de cuidado à pessoa com diabetes.

Como elementos da SAE, foi mencionado pelos enfermeiros a realização do exame físico e avaliação da pele, no entanto, não foi considerado o exame do pé diabético conforme o recomendado nas diretrizes do Ministério da Saúde (Brasil, 2016). O exame do pé não é visto como parte do exame físico a ser realizado pelo enfermeiro no cenário hospitalar por falta de materiais, e, por entender ser uma das práticas da atenção primária (Barz et al., 2019).

A coordenação do cuidado ao exame do pé é responsabilidade da atenção primaria, porém essa deve acompanhar o indivíduo com diabetes dentro do Sistema de Saúde, incluindo os demais níveis de atenção, assim como, promover a articulação entre eles. Para isso, é indispensável uma comunicação efetiva entre os níveis de assistência, que pode ser realizado pela comunicação direta com ligações telefônicas (Brasil, 2016). Este achado aponta para a necessidade de enfermeiros incluírem no processo de cuidar, no histórico de enfermagem, como parte do exame físico, o exame do pé diabético, assim como qualifiquarem as orientações durante a internação e, para a alta hospitalar sobre o cuidado a ser desenvolvido para prevenir complicações e, 
articular com a atenção básica para contra referência.

As atividades assistenciais foram elencadas como práticas de cuidado, por possibilitarem ao enfermeiro intervir e avaliar, construir um espaço de escuta e diálogo, confiança e vínculo, na interação profissional enfermeiro-paciente. É nestes encontros da realização de práticas técnicas/procedimentos de enfermagem que essa interação se intensifica, informações são compartilhadas e, o vínculo poderá ser potencializado. Este processo resulta em importantes dispositivos mobilizados na pessoa com diabetes, como promoção da autonomia, autoestima e empoderamento para gerenciar o tratamento e tomar decisões. Além disso, é nestes encontros que o enfermeiro direciona o olhar à família, assim conhece e avalia a estrutura, funcionalidade, fase do ciclo de vida e, identifica o cuidador familiar. Entre as atividades assistenciais que se destacaram foi o manejo e realização do controle de glicêmica capitalar e, práticas voltadas a prevenir lesões cutâneas.

É crescente há a necessidade de uma "prática de enfermagem nesse contexto, com uma visão mais ampla, que inclua as pessoas de forma holística, com um protagonismo e empoderamento de câmbio, capazes de autocontrolar sua saúde, envolvendo a sua família e rede social nessa experiência de vida" (Dávila, Acelas \& Montañez, 2019, p.3). O encontro do enfermeiro com paciente e familiar, trata-se de um encontro entre o cuidado técnico com expressivo e educativo. O ensinar como um processo transversal do cuidado de enfermagem e, que pode se intensificar quando o enfermeiro realiza determinado procedimento técnico.

No presente estudo identificou-se que há uma preocupação dos enfermeiros em desenvolver ações de educação em saúde no hospital para pacientes com diabetes. Estas ações foram desenvolvidas de forma individual com o paciente, e com a família, familiar cuidador; mediante conversas sobre alimentação, o controle da glicemia, cuidados com insulina, cuidado com os pés e como evitar lesões cutâneas. Estas ações tinham como finalidade proporcionar orientações de continuidade do cuidado no domicílio

Os pacientes com diabetes possuem déficit de conhecimento para realizar o controle da glicêmica capilar, uma vez que, tem dificuldades em manusear o aparelho, não conseguem interpretar valores e, não o registram (Oliveira et al., 2018). Estudo evidenciou que 77,9\% desses pacientes apresentam risco para desenvolver lesões (Damas-Casani, Yovera-Aldana \& Santisteban, 2017), sinalizando este grupo como de maior vulnerabilidade no hospital. Tais achados necessitam ser considerados pelo enfermeiro no planejamento de práticas de cuidado. O profissional de saúde precisa intervir e, orientar às pessoas com diabetes, quanto a importância da utilização e, monitoramento da glicemia capilar, assim como ensiná-lo para uso posterior no domicílio (Oliveira et al., 2018).

O hospital vem sendo descrito como importante espaço para promover ações de educação em saúde incluindo a implementação de grupos (Arruda \& Silva, 2020). A educação em saúde não está desvinculada dos cuidados técnicos desenvolvidos neste cenário (Acioli et al., 2014; Castro-Meza, Pérez-Zumano \&Salcedo-Álvarez, 2017), tanto que o momento da realização do histórico de enfermagem foi reconhecido como ideal para iniciar o cuidado educativo (Arruda \& Silva, 2020).

Os enfermeiros proporcionam informações, mediante orientações e recomendações, tanto para paciente com diabetes quanto aos familiares (Arruda \& Silva, 2020; Castro-Meza, Pérez-Zumano \&Salcedo-Álvarez, 2017), as quais correspondem a alimentação, cuidado com os pés, manuseio do glicosímetro e realização de glicemia capitalar a fim de evitar complicações, prepará-los para retorno ao domicílio, ajudar e fomentar a participação para tomada de decisão sobre sua condição e atenção (Castro-Meza, Pérez-Zumano \& Salcedo-Álvarez, 2017). Ainda o estudo aponta que não há um momento específico para ensinar, sendo este definido como a ação em que a enfermeira e a pessoa se conectam em um momento, em que ao mesmo tempo em que se realiza um cuidado se ensina a forma de realizar seu próprio cuidado (Castro-Meza, Pérez-Zumano \& Salcedo-Álvarez, 2017).

No entanto, para realização de práticas de educação em saúde no hospital os enfermeiros enfrentam diversos obstáculos, entre eles o número insuficiente de profissionais da saúde, principalmente de enfermagem, a elevada demanda de trabalho (assistencial e de gestão); além do espaço no hospital, deficiência de ambientes como salas, para atividades coletivas e, a deficiência de materiais como vídeos, cartilhas ou folders (Arruda \& Silva, 2020). Algumas possibilidades apontadas para o 
enfermeiro desenvolver educação em saúde no hospital são, a necessidade de recursos didáticos com o uso de materiais lúdicos educativos e uso de instrumentos específicos para registros da realização da educação em saúde pela equipe de enfermagem (Arruda \& Silva, 2020).

\section{Considerações Finais}

A construção deste estudo possibilitou descrever as práticas de cuidado realizadas por enfermeiros à pacientes com diabetes mellitus. Identificou-se que as práticas desenvolvidas a estes pacientes se referem ao controle da glicemia, cuidados com a alimentação, com a pele e pés a fim de prevenir lesões cutâneas. Realizam práticas voltadas para recuperação, promoção da saúde e, prevenção de complicações, com a finalidade de mobilizá-los para o autocuidado a partir de proporcionar orientações e recomendações à pessoa com diabetes e aos familiares.

Os resultados deste estudo não podem ser generalizados, uma vez que, o universo dos enfermeiros no cenário deste hospital, está inserido em rede de atenção à saúde, a cultura do local, e as interações que são construídas de acordo com a gestão da instituição. Como limitação o fato de o estudo ter sido realizado somente em um hospital, sendo este de ensino, e ao utilizar uma única técnica de coleta, uma vez que a inclusão de outras técnicas poderia possibilitar que dados fossem aprofundados em outros momentos, outras práticas de cuidado teriam sido identificadas, as quais podem não ter sido mencionadas nas entrevistas semiestruturadas.

A partir deste estudo constata-se a necessidade de desenvolver outras pesquisas que aprofundem as práticas de cuidado a pessoas com diabetes no hospital, considerando a perspectiva dos pacientes, dos familiares e dos demais profissionais envolvidos nesse processo. Estudos que explorem a educação em saúde como dimensão do cuidar, e estudos sobre a contra referência destes pacientes na rede de atenção a saúde e, articulação entre os níveis de atenção.

\section{Referências}

Acioli S., Kebian L. V. A., Faria M. G. A., Ferraccioli P. \& Correa V. A. F. (2014). Práticas de cuidado: o papel do enfermeiro na atenção básica. Revista Enfermagem UERJ, 22(5):637-42. https://doi.org/10.12957/reuerj.2014.12338

Aguayo-Verdugo N. V. \& Orellana-Yañez A. E. (2019). Intervenciones de enfermería en pacientes con diabetes mellitus tipo 2 que aumentan sus conductas de autocuidado: revisión integrativa. Enfermería Actual de Costa Rica, (36):116-29. http://dx.doi.org/10.15517/revenf.v0i36.34238

Arruda C. \& Silva D. M. G. V. (2020). A hospitalização como espaço para educação em saúde às pessoas com diabetes mellitus. Rev Fun Care Online, 12:3745. http://dx.doi.org/10.9789/2175-5361.rpcfo. v12.6909

Bardin L. (2016). Análise de Conteúdo. Edições 70.

Barz D. B., Barcellos C. R. B., Genezi C., Peters C., Schwanck D. C. \& Zillmer J. G. V. (2019). Vivências de acadêmicos de enfermagem na avaliação e aplicação do exame do pé à pessoa com diabetes: relato de experiência. V Congresso de Iniciação Científica. Semana Integrada da Universidade Federal de Pelotas. https://cti.ufpel.edu.br/siepe/arquivos/2019/CS_01158.pdf

Bourdieu P. (1994). Esboço de uma teoria da prática. In: Ortiz R, organizador. Pierre Bourdieu: sociologia. Ática. p. 46-81.

Brasil (2016). Ministério da Saúde.. Manual do Pé Diabético, Estratégias para o Cuidado da Pessoa com Doença Crônica. http://189.28.128.100/dab/docs/portaldab/publicacoes/manual_do_pe_diabetico.pdf

Carvalho S. L., Ferreira M. A., Medeiros J. M. P., Queiroga A. C. F., Moreira T. R. \& Negreiros F. D. S. (2018). Conversation map: an educational strategy in the care of elderly people with diabetes mellitus. Rev Bras Enferm. Rev. Bras. Enferm. 71(Suppl2):925-929. https://doi.org/10.1590/0034-7167-2017-0064

Castro-Meza A. N., Pérez-Zumano S. E., Salcedo-Álvarez R. A., Castro-Meza A. N., Pérez-Zumano S. E. \& Salcedo-Álvarez R. A. (2017). La enseñanza a pacientes con diabetes: significado para profesionales de enfermería. Enfermería universitária, 14(1):39-46. https://doi.org/10.1016/j.reu.2016.12.003

Cofen (2009). Conselho Federal de Enfermagem. Resolução COFEN n 358/2009. http://www.cofen.gov.br/resoluo-cofen-3582009_4384.html

Damas-Casani V. A., Yovera-Aldana M. \& Seclén Santisteban S. (2017). Clasificación de pie en riesgo de ulceración según el Sistema IWGDF y factores asociados en pacientes con diabetes mellitus tipo 2 de un hospital peruano. Rev Med Hered. 28(1):5-12. http://dx.doi.org/10.20453/rmh.v28i1.3067

Dávila C. M. G., Acelas A. L. R. \& Montañez W. C. (2019). Soporte social enfocado a personas con diabetes: una necesidad desde enfermería. Rev Cuid.10(1). https://doi.org/10.15649/cuidarte.v10i1.697 
Research, Society and Development, v. 10, n. 15, e472101522779, 2021

(CC BY 4.0) | ISSN 2525-3409 | DOI: http://dx.doi.org/10.33448/rsd-v10i15.22779

Flor L. S. \& Campos M. R. (2017). Prevalência de diabetes mellitus e fatores associados na população adulta brasileira: evidências de um inquérito de base populacional. Rev. bras. epidemiol. [Internet]. 20(1):16-29. https://doi.org/10.1590/1980-5497201700010002

Minayo M. C. S. (2017). Amostragem e saturação em pesquisa qualitativa: consensos e controvérsias. Revista Pesquisa Qualitativa, 5(7):01-12. https://editora.sepq.org.br/index.php/rpq/article/view/82/59

Mwila K. F., Bwembya P. A. \& Jacobs C. (2019). Experiences and challenges of adults living with type 2 diabetes mellitus presenting at the University Teaching Hospital in Lusaka, Zambia. BMJ Open Diab Res Care, 7(1):e000497. http://dx.doi.org/10.1136/bmjdrc-2017-000497

Nogueira L. G. F., Medeiros A. C. T., Bittencourt G. K. G. D \& Nóbrega M. M. L. (2016). Diagnósticos, resultados e intervenções de enfermagem ao idoso diabético: estudo de caso. Online braz j nurs. 15 (2):302-312. http://www.objnursing.uff.br/index.php/nursing/article/view/4964

Oliveira R. F., Barbosa J. A. G., Guimarães G. L., Silva S. M., Andrade M. I. \& Calsolari M. R. (2018). Automonitorização glicêmica: Dificuldades na realização do procedimento por pacientes com diabetes mellitus. Rev Min Enferm. 22:e-1117. http://www.dx.doi.org/10.5935/1415-2762.20180052

Pesantes M. A., Valle A. D., Diez-Canseco F., Bernabé-Ortiz A., Portocarrero J., Trujillo A., . . Miranda J. J. (2018). Family support and diabetes: patient's experiences from a public hospital in Peru. Qual Health Res. 28(12):1871-1882. https://doi.org/10.1177/1049732318784906

Salci M. A., Meirelles B. H. S. \& Silva D. M. G. V. (2018). Health education to prevent chronic diabetes mellitus complications in primary care. Esc. Anna Nery. 22(1):e20170262. https://doi.org/10.1590/2177-9465-ean-2017-0262

Silva L. W. S., Silva J. S., Squarcini C. F. R., Souza F. G., Ribeiro V. S. \& Gonçalves D. F. (2016). Promoção da saúde de pessoas com diabetes mellitus no cuidado educativo preventivo do pé-diabético. Cienc. enferm. 22(2):103-116. http://dx.doi.org/10.4067/S0717-95532016000200008.

Soares M. I., Resck Z. M. R., Terra F. S. \& Camelo S. H. H. (2015). Sistematização da assistência de enfermagem: facilidades e desafios do enfermeiro na gerência da assistência. Esc. Anna Nery. 19(1):47-53. https://doi.org/10.5935/1414-8145.20150007.

Sociedade Brasileira de Diabetes (2017). Diretrizes Sociedade Brasileira de Diabetes 2017-2018. https://www.diabetes.org.br/profissionais/images/2017/diretrizes/diretrizes-sbd-2017-2018.pdf

Teston E. F., Spigolon D. N., Maran E., Santos A. L., Matsuda L. M. \& Marcon S. S. (2018). Perspectiva de enfermeiros sobre educação para a saúde no cuidado com o diabetes mellitus. Rev. Bras. Enferm. 71:2735- 2742. https://doi.org/10.1590/0034-7167-2018-0396

Zillmer J. G. V., Schwartz E. \& Muniz R. M. (2012). O olhar da enfermagem sobre as práticas de cuidado de famílias rurais à pessoa com câncer. Rev. esc. enferm. USP. 46(6):1371-8. https://doi.org/10.1590/S0080-62342012000600013 